INPLASY

PROTOCOL

To cite: Luo et al. Acupoint catgut embedding reduces postoperative pain of mixed hemorrhoids: a meta-analysis of randomized controlled trials. Inplasy protocol 202220021. doi:

10.37766/inplasy2022.2.0021

Received: 09 February 2022

Published: 09 February 2022

Corresponding author: Jing Luo

luojingne@163.com

Author Affiliation:

TaiZhou Traditional Chinese Medicine Hospital.

Support: None.

Review Stage at time of this submission: Data analysis Completed but not published.

\section{Acupoint catgut embedding reduces postoperative pain of mixed hemorrhoids: a meta-analysis of randomized controlled trials}

\author{
Luo, J1; Zhang, C2; Xia, M33 Chen, Y4.
}

Review question / Objective: Can acupoint catgut embedding alleviate postoperative pain of mixed hemorrhoids?

Condition being studied: Mixed hemorrhoids.

Information sources: Two authors (JL and CZ) will examine the publications independently and extract data according to predefined criteria. RCTs will be assessed for the methodology, study design, inclusion and exclusion criteria, and outcome measures. The methodological quality of each RCT will be recorded for method of randomization, blinding, protocol violation, and allocation concealment Any disagreement will be resolved by consensus discussions with the senior member of the review team (MJX and YLC). Data to collect includes intervention and control measures, measured outcomes and statistical significant difference with regards to chewing gum.

INPLASY registration number: This protocol was registered with the International Platform of Registered Systematic Review and Meta-Analysis Protocols (INPLASY) on 09 February 2022 and was last updated on 09 February 2022 (registration number INPLASY202220021).

Conflicts of interest:

None declared.

\section{INTRODUCTION}

Review question / Objective: Can acupoint catgut embedding alleviate postoperative pain of mixed hemorrhoids?

Condition being studied: Mixed hemorrhoids.

\section{METHODS}

Search strategy: Mixed hemorrhoids AND A cupoint Catgut Embedding search strategy \#1 "Hemorrhoids"[Mesh]

\#2 "Hemorrhoid"[Title / Abstract] OR "Mixed hemorrhoids"[Title / Abstract] \#3 \#1 OR \#2 
\#4 "Acupuncture"[MeSH]

OR "acupotomy" [Title/Abstract]OR "acupotomies"[Title/Abstract] OR "acupoint" [Title/Abstract]OR "acupoint catgut embedding" [Title/Abstract] OR "acupoint thread embedding"[Title/ Abstract] OR "catgut implantation" [Title/ Abstract] OR "acupoints thread embedding"[Title/Abstract]OR "catgu-t embedding" [ Title/Abstract] OR "embedding therapy"[Title/Abstract] OR "thread-embedding" [Title/Abstract] \#5\#3 AND \#4

\#6 "Analgesia" [MeSH]

OR "analgesi"” [Title/Abstract] OR "pain*" [Title/Abstract]OR "relie*" [Title/Abstract] OR "sufentanil" [Title/Abstract] OR "fentanyl" [Title/Abstract] OR "Iornoxicam" [Title/Abstract] OR "tramadol" [Title/ Abstract] OR "ketamine" [Title/Abstract] OR "flurbiprofen"[Title/Abstract] OR "morphine" [Title/Abstract] OR "morphia"[Title/Abstract] OR "morphina " \#7 \#5 AND \#6.

Participant or population: Patients who with mixed hemorrhoids hyperlipidemia.

Intervention: Acupoint Catgut Embedding.

Comparator: Analgesic.

Study designs to be included: RCT.

Eligibility criteria: Cohort studies and other studies were excluded.

Information sources: Two authors (JL and CZ) will examine the publications independently and extract data according to predefined criteria. RCTs will be assessed for the methodology, study design, inclusion and exclusion criteria, and outcome measures. The methodological quality of each RCT will be recorded for method of randomization, blinding, protocol violation, and allocation concealment Any disagreement will be resolved by consensus discussions with the senior member of the review team (MJX and $Y L C)$. Data to collect includes intervention and control measures, measured outcomes and statistical significant difference with regards to chewing gum.

Main outcome(s): The relationship between mixed hemorrhoids and acupoint catgut embedding.

Additional outcome(s): None.

Quality assessment / Risk of bias analysis: Any disagreement will be resolved by consensus discussions with the senior member of the review team (MJXand YLC).

Strategy of data synthesis: Using the standard method recommended by the Cochrane Collaboration, the outcome index included in the meta-analysis was pain. The random-effect model was predetermined to be most suitable for this meta-analysis, considering both catgut embedding and analgesia.

Subgroup analysis: Comparison of different points and drug method.

Sensitivity analysis: Have sensitivity analysis.

Language: English.

Country(ies) involved: China.

Keywords: acupoint catgut embedding, Analgesic, Mixed hemorrhoids.

Contributions of each author:

Author 1 - Jing Luo.

Email: luojingne@163.com

Author 2 - Chen Zhang.

Email: chan299@163.com

Author 3 - Mengjie Xia.

Email: e-xmj1140590151@outlook.com

Author 4 - Yuelian Chen.

Email: 103618894@qq.com 